\title{
Lista das espécies de Pyrgotidae (Diptera, Tephritoidea) do Estado do Mato Grosso do Sul
}

\author{
Ramon Luciano Mello
}

Laboratório de Sistemática de Diptera, Universidade Federal de Mato Grosso do Sul, Caixa Postal 549, 79070-900, Campo Grande, MS, Brasil. (ramonlmello@gmail.com)

Recebido 30 novembro 2016

Aceito 6 fevereiro 2017

DOI: $10.1590 / 1678-4766 e 2017145$

ABSTRACT. Checklist of the species of Pyrgotidae (Diptera, Tephritoidea) from Mato Grosso do Sul State. In this paper is presented an updated checklist of the species of Pyrgotidae (Diptera, Tephritoidea) distributed in the Mato Grosso do Sul state. The fauna of Pyrgotidae is represented by six species in four genera with distribution restricted to the Pantanal Biome.

KEYWORDS. Pyrgotinae, Scarabaeidae, Pantanal, Biota-MS Program

RESUMO. Neste artigo é apresentada uma lista atualizada das espécies de Pyrgotidae (Diptera, Tephritoidea) encontradas no estado do Mato Grosso do Sul. A fauna de Pyrgotidae do Mato Grosso do Sul é representada por seis espécies, em quatro gêneros, com distribuição restrita ao Bioma do Pantanal.

PALAVRAS-CHAVE. Pyrgotinae, Scarabaeidae, Pantanal, Programa Biota-MS.

A família Pyrgotidae apresenta distribuição mundial, integrando cerca de 370 espécies em 55 gêneros (KORNEYEV, 2006). Segundo Mello (2014) na América do Sul são conhecidas 52 espécies dispostas em 11 gêneros. O Brasil apresenta a maior biodiversidade de Pyrgotidae na Região Neotropical, com 35 espécies registradas até o momento, sendo uma das maiores do mundo.

As larvas da família são endoparasitas abdominais de coleópteros pertencentes à família Scarabaeidae. Mello et al. (2010) identificaram Pelidnota sordida Germar (Coleoptera, Scarabaeidae) como a espécie hospedeira de Carrerapyrgota bernardii Mello, Lamas \& Rafael, 2010. Esse foi o primeiro registro de uma espécie hospedeira para a família no Brasil. Assim como seus hospedeiros, os pirgotídeos apresentam hábitos noturnos, sendo comumente capturados durante a noite por meio de atrativo luminoso.

A família apresenta representantes com porte médio a grande, comprimento do corpo, sem a antena, varia entre 4,0 - 30,0 mm. A coloração do corpo, na maioria das espécies, é castanha, podendo variar entre o amarelo, castanho-avermelhado, marrom ou negro. A asa é hialina ou possui coloração castanha e, em geral, apresenta padrões de manchas distintos.

\section{MATERIAL E MÉTODOS}

A lista das espécies de Pyrgotidae do estado do Mato Grosso do Sul foi elaborada com base na revisão das espécies de Pyrgotidae do Novo Mundo, resultante da tese de doutorado do autor (R. L. Mello, dados inéditos). Para a elaboração da lista, foram consideradas somente as modificações taxonômicas já publicadas.

\section{RESULTADOS E DISCUSSÃO}

Lista das espécies de Pyrgotidae do Estado do Mato Grosso do Sul

Carrerapyrgota Aczél, 1956

Carrerapyrgota miliaria Aczél, 1956. Localidade-tipo:

Salobra, Mato Grosso do Sul, Brasil. Ocorrência no estado:

Salobra (localidade-tipo).

Carrerapyrgota personata (Lutz \& Lima, 1918).

Localidade-tipo: Palmares, Pernambuco, Brasil.

Ocorrência no estado: Salobra.

$$
\text { Idiopyrgota Aczél, } 1956
$$

Idiopyrgota setiventris Aczél, 1956. Localidade-tipo: Salobra, Mato Grosso do Sul, Brasil. Ocorrência no estado: Salobra (localidade-tipo).

$$
\text { Neopyrgota Hendel, } 1934
$$

Neopyrgota (Allopyrgota) hennigi Aczél, 1956. Localidadetipo: Campos do Jordão, São Paulo, Brasil. Ocorrência no estado: Corumbá.

$$
\text { Pyrgota Wiedemann, } 1830
$$

Pyrgota ilona Aczél, 1956. Localidade-tipo: Agua Blanca, Salta, Argentina. Ocorrência no estado: Corumbá e Salobra. Pyrgota longipes Hendel, 1908. Localidade-tipo: Rio Grande do Sul, Brasil. Ocorrência no estado: Salobra. 
Comentários à lista de distribuição. As ocorrências de Pyrgotidae no Estado do Mato Grosso do Sul estão restritas ao oeste do estado, nos municípios de Corumbá e Salobra, região correspondente ao Bioma Pantanal. O município de Salobra é a localidade-tipo das espécies Carrerapyrgota miliaria e Idiopyrgota setiventris.

A maioria dos espécimes de pirgotídeos coletados no Estado é proveniente das coletas realizadas nos anos de 1938 e 1940, pela Comissão do Instituto Oswaldo Cruz (Com. I.O.C). Embora a maior parte dos exemplares provenha de coletas realizadas pela Com. I.O.C, a principal instituição depositária desse acervo é a coleção do Museu de Zoologia da Universidade de São Paulo (MZUSP).

Principais grupos de pesquisa e acervos. O principal grupo de pesquisa em sistemática e taxonomia de Pyrgotidae no Brasil encontra-se atualmente na Universidade Federal do Mato Grosso do Sul, sob a coordenação do Dr. Ramon Luciano Mello.

Com base nos dados levantados por R. L. Mello (dados inéditos), as instituições detentoras de acervo de Pyrgotidae no Brasil são: Departamento de Zoologia da Universidade Federal do Paraná (DZUP); Fundação e Instituto Oswaldo Cruz (FIOCRUZ); Instituto Nacional de Pesquisas da Amazônia (INPA); Museu Nacional do Rio de Janeiro (MNRJ) e Museu de Zoologia da Universidade de São Paulo (MZUSP).

Perspectivas de pesquisa para os próximos dez anos. A partir das coletas resultantes do projeto "Diptera dos estados do Mato Grosso, Mato Grosso do Sul e Rondônia: diversidade, sistemática e limites distribucionais" esperase um aumento no número de espécies registradas para o Estado, bem como, no número de localidades em que essas espécies se distribuem.

Agradecimentos. A Fundação de Apoio ao Desenvolvimento do Ensino, Ciências e Tecnologia do Estado de Mato Grosso do Sul (Fundect) e a Superintendência de Ciências e Tecnologia do Estado de Mato Grosso do Sul (Sucitec/MS) pelo convite de participação neste fascículo especial da Iheringia, Série Zoologia e o suporte financeiro para sua publicação. Ao Dr. Carlos Lamas (MZUSP) e Dra. Vera Cristina Silva (UNESP, Jaboticabal) pelos importantes comentários em uma primeira versão deste manuscrito. Ao Conselho Nacional de Desenvolvimento Científico e Tecnológico (CNPq) (Proc. 563256/2010-9) e Fundação de Amparo à Pesquisa do Estado de São Paulo (FAPESP) (Proc. 2010/52314-0) pelo auxílio conferido ao projeto "Rede temática para estudos de diversidade, sistemática e limites distribucionais de Diptera nos estados do Mato Grosso, Mato Grosso do Sul e Rondônia", aprovado no âmbito do Edital SISBIOTA.

\section{REFERÊNCIAS BIBLIOGRÁFICAS}

Korneyev, V. 2006. A revision of Afrotropical species of the Eupyrgota (Diptera, Pyrgotidae): the spinifemur group and latipennis subgroup of species. Vestnik Zoologii 40:3-25.

Mello, R. L. 2011. Sistemática de Pyrgotidae do Novo Mundo (Diptera, Schizophora). Tese de doutorado. São Paulo, Universidade de São Paulo.

Mello, R. L. 2014. Pyrgotidae. In: Roig-Juñet, S.; Claps L. E. \& Morrone, J. J. eds. Biodiversidad de Artrópodos Argentinos. 1ed. San Miguel de Tucumán: INSUE - Universidad Nacional de Tucumán, 2014, v. 4, p. 499-503.

Mello, R. L.; Lamas. C. J. E. \& Rafael, J. A. 2010. Revision of the Neotropical genus Carrerapyrgota Aczél (Diptera, Pyrgotidae) with the description of two new species. Zootaxa 2515:45-64. 